\title{
A numerical investigation on the structure of the zeros of the degenerate Euler-tangent mixed-type polynomials
}

\author{
Cheon Seoung Ryoo \\ Department of Mathematics, Hannam University, Daejeon 306-791, Korea.
}

Communicated by A. Atangana

\begin{abstract}
In this paper, we obtain a general symmetric identity involving the degenerate Euler-tangent mixed-type polynomials and sums of generalized falling factorials. We use this identity to describe some combinatorial relations between these polynomials and generalized factorial alternating sums. Finally, we observe an interesting phenomenon of "scattering" of the zeros of degenerate Euler-tangent mixed-type polynomials. (C)2017 All rights reserved.
\end{abstract}

Keywords: Degenerate Euler polynomials, degenerate tangent polynomials, degenerate Euler-tangent mixed-type polynomials, generalized falling factorials, generalized factorial alternating sums, Stirling numbers of the first kind. 2010 MSC: 11B68, 11S40, 11S80.

\section{Introduction}

Computing environment would make more and more rapid progress and there has been increasing interest in solving mathematical problems with the aid of computers. Using software, mathematicians can explore concepts much more easily than in the past. The ability to create and manipulate figures on the computer screen enables mathematicians to quickly visualize and produce many problems, examine properties of the figures, look for patterns, and make conjectures. This capability is especially exciting because these steps are essential for most mathematicians to truly understand even basic concept. Numerical experiments of Bernoulli polynomials, Euler polynomials, and Genocchi polynomials have been the subject of extensive study in recent years and much progress has been made both mathematically and computationally (see [1-6, 8-11]). In [2], Carlitz introduced the degenerate Bernoulli polynomials. Recently, Qi et al. [6] studied the partially degenerate Bernoull polynomials of the first kind in p-adic field.

Throughout this paper, we always make use of the following notations: $\mathbb{N}$ denotes the set of natural numbers, $\mathbb{Z}_{+}=\mathbb{N} \cup\{0\}$, and $\mathbb{C}$ denotes the set of complex numbers. Ryoo [11] defined the degenerate tangent polynomials $T_{n, \lambda}(x)$ for $\lambda \neq 0$ by means of the generating function

$$
\frac{2}{(1+\lambda t)^{2 / \lambda}+1}(1+\lambda t)^{x / \lambda}=\sum_{n=0}^{\infty} \mathcal{T}_{n, \lambda}(x) \frac{t^{n}}{n !} .
$$

Email address: ryoocs@hnu.kr (Cheon Seoung Ryoo)

doi:10.22436/jnsa.010.08.39 
When $x=0, \mathcal{T}_{n, \lambda}(0)=\mathcal{T}_{n, \lambda}$ are called the degenerate tangent numbers. For more theoretical properties of the degenerate tangent numbers and polynomials, the readers may refer to [11]. These are polynomials in $x$ and $\lambda$ with integer coefficients (see [11]). We recall that the classical Stirling numbers of the first kind $S_{1}(n, k)$ and $S_{2}(n, k)$ are defined by the relations (see [12])

$$
(x)_{n}=\sum_{k=0}^{n} s_{1}(n, k) x^{k} \text { and } x^{n}=\sum_{k=0}^{n} S_{2}(n, k)(x)_{k},
$$

respectively. The generalized falling factorial $(x \mid \lambda)_{n}$ with increment $\lambda$ is defined by

$$
(x \mid \lambda)_{n}=\prod_{k=0}^{n-1}(x-\lambda k)
$$

for positive integer $n$, with the convention $(x \mid \lambda)_{0}=1$. We also need the binomial theorem: for a variable $x$,

$$
(1+\lambda t)^{x / \lambda}=\sum_{n=0}^{\infty}(x \mid \lambda)_{n} \frac{t^{n}}{n !} .
$$

The tangent numbers $T_{n}$ are defined by means of the generating function (see, for details, [9]):

$$
\sum_{n=0}^{\infty} T_{n} \frac{t^{n}}{n !}=\frac{2}{e^{2 t}+1}, \quad\left(|t|<\frac{\pi}{2}\right) .
$$

The second kind Euler polynomials $E_{n}(x)$ are defined by the generating function (see, for details, [8]):

$$
\left(\frac{2 e^{t}}{e^{2 t}+1}\right) e^{x t}=\sum_{n=0}^{\infty} E_{n}(x) \frac{t^{n}}{n !} .
$$

We also define the second kind degenerate kind Euler polynomials $\mathcal{E}_{n, \lambda}(x)$ by

$$
\frac{2(1+\lambda t)^{1 / \lambda}}{(1+\lambda t)^{2 / \lambda}+1}(1+\lambda t)^{x / \lambda}=\sum_{n=0}^{\infty} \varepsilon_{n, \lambda}(x) \frac{t^{n}}{n !} .
$$

When $x=0, \varepsilon_{n, \lambda}(0)=\mathcal{E}_{n, \lambda}$ are called the second kind degenerate Euler numbers.

Using computer, a realistic study for degenerate Euler-tangent mixed type polynomials is very interesting. The outline of this paper is as follows. In Section 2, we define new degenerate Euler-tangent mixed-type polynomials $\gamma_{n, \lambda}(x)$. We give some properties of these polynomials $\gamma_{n, \lambda}(x)$. In Section 3, we obtain a general symmetric identity involving the degenerate tangent polynomials and sums of generalized falling factorials. In Section 4, we observe the structure of the real roots of our polynomials, $\gamma_{n, \lambda}(x)$, using numerical investigation. By computer experiments, we demonstrate a remarkably regular structure of the complex roots of the Euler-tangent mixed type polynomials $\gamma_{n, \lambda}(x)$.

\section{New degenerate Euler-tangent mixed-type polynomials}

In this section, we define the degenerate Euler-tangent-type polynomials $\gamma_{n, \lambda}(x)$. Our main identity in Section 3 will be derived from the degenerate Euler-tangent mixed-type polynomials. For a variable $t$, the degenerate Euler-tangent mixed-type polynomials $\gamma_{n, \lambda}(x)$ are defined by means of the generating function:

$$
\frac{2\left((1+\lambda t)^{(x+1) / \lambda}+1\right)}{(1+\lambda t)^{2 / \lambda}+1}=\sum_{n=0}^{\infty} \gamma_{n, \lambda}(x) \frac{t^{n}}{n !}
$$


When $x=0, \gamma_{n, \lambda}(0)=\gamma_{n, \lambda}$ are called the degenerate Euler-tangent mixed-type numbers. Note that $\gamma_{n, \lambda}(x)=\mathcal{E}_{n, \lambda}(x)+\mathcal{T}_{n, \lambda}$. These are polynomials in $x$ and $\lambda$ with integer coefficients. From (2.1), (1.3), and (1.4), we note that

$$
\sum_{n=0}^{\infty} \lim _{\lambda \rightarrow 0} \gamma_{n, \lambda}(x) \frac{t^{n}}{n !}=\lim _{\lambda \rightarrow 0} \frac{2\left((1+\lambda t)^{(x+1) / \lambda}+1\right)}{(1+\lambda t)^{2 / \lambda}+1}=\left(\frac{2 e^{t}}{e^{2 t}+1}\right) e^{x t}+\frac{2}{e^{2 t}+1}=\sum_{n=0}^{\infty}\left(E_{n}(x)+T_{n}\right) \frac{t^{n}}{n !} .
$$

Thus, we get

$$
\lim _{\lambda \rightarrow 0} \gamma_{n, \lambda}(x)=E_{n}(x)+T_{n},(n \geqslant 0) .
$$

By (2.1), we get

$$
\begin{aligned}
\sum_{n=0}^{\infty} \gamma_{n,-\lambda}(-x-2) \frac{(-t)^{n}}{n !} & =\frac{2\left((1+\lambda t)^{(x+1) / \lambda}+1\right)}{(1+\lambda t)^{2 / \lambda}+1}(1+\lambda t)^{2 / \lambda} \\
& =\left(\sum_{n=0}^{\infty} \gamma_{n, \lambda}(x) \frac{t^{n}}{n !}\right)\left(\sum_{n=0}^{\infty}(2 \mid \lambda)_{n} \frac{t^{n}}{n !}\right) \\
& =\sum_{n=0}^{\infty}\left(\sum_{l=0}^{n}\left(\begin{array}{l}
n \\
l
\end{array}\right) \gamma_{l, \lambda}(x)(2 \mid \lambda)_{n-l}\right) \frac{t^{n}}{n !}
\end{aligned}
$$

By comparing of the coefficients $\frac{t^{n}}{n !}$ on the both sides of (2.2), we have the following theorem.

Theorem 2.1. For $\mathrm{n} \in \mathbb{Z}_{+}$, we have

$$
\gamma_{n,-\lambda}(-x-2)=(-1)^{n} \sum_{l=0}^{n}\left(\begin{array}{l}
n \\
l
\end{array}\right) \gamma_{l, \lambda}(x)(2 \mid \lambda)_{n-l} .
$$

By (2.1) and (1.1), we have

$$
\begin{aligned}
\sum_{n=0}^{\infty}\left(\mathcal{T}_{n, \lambda}(x+1)+\mathcal{T}_{n, \lambda}\right) \frac{t^{n}}{n !} & =\frac{2}{(1+\lambda t)^{2 / \lambda}+1}(1+\lambda t)^{(x+1) / \lambda}+\frac{2}{(1+\lambda t)^{2 / \lambda}+1} \\
& =\frac{2\left((1+\lambda t)^{(x+1) / \lambda}+1\right)}{(1+\lambda t)^{2 / \lambda}+1} \\
& =\sum_{m=0}^{\infty} \gamma_{m, \lambda}(x) \frac{t^{m}}{m !} .
\end{aligned}
$$

By comparing of the coefficients $\frac{t^{n}}{n !}$ on the both sides of (2.3), we have the following theorem.

Theorem 2.2. For $\mathrm{n} \in \mathbb{Z}_{+}$, we have

$$
\mathcal{T}_{n, \lambda}(x+1)+\mathcal{T}_{n, \lambda}=\gamma_{n, \lambda}(x) .
$$

For $n \in \mathbb{N}$ with $n \equiv 1(\bmod 2)$, we have

$$
\begin{aligned}
\sum_{m=0}^{\infty}\left(\mathcal{T}_{m, \lambda}(2 n)+\mathcal{T}_{m, \lambda}\right) \frac{t^{m}}{m !} & =\frac{2}{(1+\lambda t)^{2 / \lambda}+1}(1+\lambda t)^{(2 n) / \lambda}+\frac{2}{(1+\lambda t)^{2 / \lambda}+1} \\
& =\frac{2\left((1+\lambda t)^{(2 n) / \lambda}+1\right)}{(1+\lambda t)^{2 / \lambda}+1}=\sum_{m=0}^{\infty} \gamma_{m, \lambda}(2 n-1) \frac{t^{m}}{m !}
\end{aligned}
$$

and

$$
\frac{2\left((1+\lambda t)^{(2 n) / \lambda}+1\right)}{(1+\lambda t)^{2 / \lambda}+1}=2 \sum_{l=0}^{n-1}(-1)^{l}(1+\lambda t)^{2 l / \lambda}=\sum_{m=0}^{\infty}\left(2 \sum_{l=0}^{n-1}(-1)^{l}(2 l \mid \lambda)_{m}\right) \frac{t^{m}}{m !} .
$$

By (2.4) and (2.5), we have the following theorem. 
Theorem 2.3. For $\mathrm{n} \in \mathbb{N}$ with $\mathrm{n} \equiv 1(\bmod 2)$ and $\mathrm{m} \in \mathbb{Z}_{+}$, we have

$$
\gamma_{m, \lambda}(2 n-1)=2 \sum_{l=0}^{n-1}(-1)^{l}(2 l \mid \lambda)_{m}, \quad \mathcal{T}_{m, \lambda}(2 n)+\mathcal{T}_{m, \lambda}=\gamma_{m, \lambda}(2 n-1) .
$$

From (2.1) and (1.2), we have

$$
\begin{aligned}
\sum_{n=0}^{\infty} \gamma_{n, \lambda}(x+y) \frac{t^{n}}{n !} & =\frac{2\left((1+\lambda t)^{(x+y+1) / \lambda}+1\right)}{(1+\lambda t)^{2 / \lambda}+1} \\
& =\frac{2}{(1+\lambda t)^{2 / \lambda}+1}(1+\lambda t)^{(x+1) / \lambda}(1+\lambda t)^{y / \lambda}+\frac{2}{(1+\lambda t)^{2 / \lambda}+1} \\
& =\left(\sum_{n=0}^{\infty} \varepsilon_{n, \lambda}(x) \frac{t^{n}}{n !}\right)\left(\sum_{n=0}^{\infty}(y \mid \lambda)_{n} \frac{t^{n}}{n !}\right)+\sum_{n=0}^{\infty} \mathcal{T}_{n, \lambda} \frac{t^{n}}{n !} \\
& =\sum_{n=0}^{\infty}\left(\sum_{l=0}^{n}\left(\begin{array}{c}
n \\
l
\end{array}\right) \mathcal{E}_{l, \lambda}(x)(y \mid \lambda)_{n-l}+\mathcal{T}_{n, \lambda}\right) \frac{t^{n}}{n !}
\end{aligned}
$$

Therefore, by (2.6), we have the following theorem.

Theorem 2.4. For $\mathrm{n} \in \mathbb{Z}_{+}$, we have

$$
\gamma_{n, \lambda}(x+y)=\sum_{l=0}^{n}\left(\begin{array}{l}
n \\
l
\end{array}\right) \mathcal{E}_{l, \lambda}(x)(y \mid \lambda)_{n-l}+\mathcal{T}_{n, \lambda} .
$$

By replacing $t$ by $\frac{e^{\lambda t}-1}{\lambda}$ in (2.1), we obtain

$$
\begin{aligned}
\frac{2 e^{t}}{e^{2 t}+1} e^{x t}+\frac{2}{e^{2 t}+1} & =\sum_{n=0}^{\infty} \gamma_{n, \lambda}(x)\left(\frac{e^{\lambda t}-1}{\lambda}\right)^{n} \frac{1}{n !} \\
& =\sum_{n=0}^{\infty} \gamma_{n, \lambda}(x) \lambda^{-n} \sum_{m=n}^{\infty} S_{2}(m, n) \lambda^{m} \frac{t^{m}}{m !} \\
& =\sum_{m=0}^{\infty}\left(\sum_{n=0}^{m} \gamma_{n, \lambda}(x) \lambda^{m-n} S_{2}(m, n)\right) \frac{t^{m}}{m !}
\end{aligned}
$$

Thus, by (2.7), (1.4), and (1.1), we have the following theorem.

Theorem 2.5. For $n \in \mathbb{Z}_{+}$, we have

$$
E_{m}(x)+T_{m}=\sum_{n=0}^{m} \gamma_{n, \lambda}(x) \lambda^{m-n} S_{2}(m, n) .
$$

By replacing $t$ by $\log (1+\lambda t)^{1 / \lambda}$ in (1.1) and (1.4), we have

$$
\begin{gathered}
\sum_{n=0}^{\infty} E_{n}(x)\left(\log (1+\lambda t)^{1 / \lambda}\right)^{n} \frac{1}{n !}+\sum_{n=0}^{\infty} T_{n}\left(\log (1+\lambda t)^{1 / \lambda}\right)^{n} \frac{1}{n !} \\
=\frac{2\left((1+\lambda t)^{(x+1) / \lambda}+1\right)}{(1+\lambda t)^{2 / \lambda}+1}=\sum_{m=0}^{\infty} \gamma_{m, \lambda}(x) \frac{t^{m}}{m !}
\end{gathered}
$$


and

$$
\begin{aligned}
& \sum_{n=0}^{\infty} E_{n}(x)\left(\log (1+\lambda t)^{1 / \lambda}\right)^{n} \frac{1}{n !}+\sum_{n=0}^{\infty} T_{n}\left(\log (1+\lambda t)^{1 / \lambda}\right)^{n} \frac{1}{n !} \\
& =\sum_{m=0}^{\infty}\left(\sum_{n=0}^{m}\left(E_{n}(x)+T_{n}\right) \lambda^{m-n} S_{1}(m, n)\right) \frac{t^{m}}{m !} .
\end{aligned}
$$

Thus, by (2.8) and (2.9), we have the following theorem.

Theorem 2.6. For $\mathrm{n} \in \mathbb{Z}_{+}$, we have

$$
\gamma_{m, \lambda}(x)=\sum_{n=0}^{m} \lambda^{m-n}\left(E_{n}(x)+T_{n}\right) S_{1}(m, n) .
$$

\section{Symmetric identity for the degenerate tangent polynomials}

In this section, we obtain a general symmetric identity involving the degenerate tangent polynomials and sums of generalized falling factorials. Let $w_{1}$ and $w_{2}$ be odd positive integers. We consider the generating function

$$
F(t, \lambda)=\frac{4(1+\lambda t)^{\frac{w_{1} w_{2} x}{\lambda}}\left((1+\lambda t)^{\frac{2 w_{1} w_{2}}{\lambda}}+1\right)}{\left((1+\lambda t)^{\frac{2 w_{1}}{\lambda}}+1\right)\left((1+\lambda t)^{\frac{2 w_{2}}{\lambda}}+1\right)} .
$$

We use (1.1) and (2.1) to expand $F(t, \lambda)$ as

$$
\begin{aligned}
F(t, \lambda) & =\frac{4(1+\lambda t)^{\frac{w_{1} w_{2} x}{\lambda}}\left((1+\lambda t)^{\frac{2 w_{1} w_{2}}{\lambda}}+1\right)}{\left((1+\lambda t)^{\frac{2 w_{1}}{\lambda}}+1\right)\left((1+\lambda t)^{\frac{2 w_{2}}{\lambda}}+1\right)} \\
& =\left(\frac{2(1+\lambda t)^{\frac{w_{1} w_{2} x}{\lambda}}}{(1+\lambda t)^{\frac{2 w_{1}}{\lambda}}}\right)\left(\frac{2\left((1+\lambda t)^{\frac{2 w_{1} w_{2}}{\lambda}}+1\right)}{(1+\lambda t)^{\frac{2 w_{2}}{\lambda}}+1}\right) \\
& =\left(\sum_{n=0}^{\infty} \mathcal{T}_{n, \lambda / w_{1}}\left(w_{2} x\right) \frac{\left(w_{1} t\right)^{n}}{n !}\right)\left(\sum_{n=0}^{\infty} \gamma_{n, \lambda / w_{2}}\left(2 w_{1}-1\right) \frac{\left(w_{2} t\right)^{n}}{n !}\right) \\
& =\sum_{n=0}^{\infty}\left(\sum_{k=0}^{n}\left(\begin{array}{l}
n \\
k
\end{array}\right) \mathcal{T}_{k, \lambda / w_{1}}\left(w_{2} x\right) w_{1}^{k} \gamma_{n-k, \lambda / w_{2}}\left(2 w_{1}-1\right) w_{2}^{n-k}\right) \frac{t^{n}}{n !} .
\end{aligned}
$$

We may also expand $F(t, \lambda)$ as

$$
\begin{aligned}
F(t, \lambda) & =\frac{4(1+\lambda t)^{\frac{w_{1} w_{2} x}{\lambda}}\left((1+\lambda t)^{\frac{2 w_{1} w_{2}}{\lambda}}+1\right)}{\left((1+\lambda t)^{\frac{2 w_{2}}{\lambda}}+1\right)\left((1+\lambda t)^{\frac{2 w_{1}}{\lambda}}+1\right)} \\
& =\left(\sum_{n=0}^{\infty} \mathcal{T}_{n, \lambda / w_{2}}\left(w_{1} x\right) \frac{\left(w_{2} t\right)^{n}}{n !}\right)\left(\sum_{n=0}^{\infty} \gamma_{n, \lambda / w_{1}}\left(2 w_{2}-1\right) \frac{\left(w_{1} t\right)^{n}}{n !}\right) \\
& =\sum_{n=0}^{\infty}\left(\sum_{k=0}^{n}\left(\begin{array}{l}
n \\
k
\end{array}\right) \mathcal{T}_{k, \lambda / w_{2}}\left(w_{1} x\right) w_{2}^{k} \gamma_{n-k, \lambda / w_{1}}\left(2 w_{2}-1\right) w_{1}^{n-k}\right) \frac{t^{n}}{n !} .
\end{aligned}
$$


By comparing coefficients $\frac{t^{n}}{n !}$ in the both sides of (3.1) and (3.2), we have the following theorem.

Theorem 3.1. Let $w_{1}$ and $w_{2}$ be odd positive integers. For $n \in \mathbb{Z}_{+}$, we have

$$
\begin{aligned}
& \sum_{k=0}^{n}\left(\begin{array}{l}
n \\
k
\end{array}\right) w_{1}^{k} w_{2}^{n-k} \mathcal{T}_{k, w_{2} \lambda}\left(w_{2} x\right) \gamma_{n-k, w_{1} \lambda}\left(2 w_{1}-1\right) \\
& \quad=\sum_{k=0}^{n}\left(\begin{array}{l}
n \\
k
\end{array}\right) w_{2}^{k} w_{1}^{n-k} \mathcal{T}_{k, w_{1} \lambda}\left(w_{1} x\right) \gamma_{n-k, w_{2} \lambda}\left(2 w_{2}-1\right) .
\end{aligned}
$$

Letting $\lambda \rightarrow 0$ in Theorem 3.1 gives the identity

$$
\sum_{k=0}^{n}\left(\begin{array}{l}
n \\
k
\end{array}\right) w_{1}^{k} w_{2}^{n-k} T_{k}\left(w_{2} x\right) \sum_{l=0}^{w_{1}-1}(-1)^{l}(2 l)^{n-k}=\sum_{k=0}^{n}\left(\begin{array}{l}
n \\
k
\end{array}\right) w_{2}^{k} w_{1}^{n-k} T_{k}\left(w_{1} x\right) \sum_{l=0}^{w_{2}-1}(-1)^{l}(2 l)^{n-k}
$$

which was proved by Ryoo ([8, Theorem 3.2]). By Theorem 3.1, we obtain the interesting symmetric identity for degenerate tangent number in complex field.

Corollary 3.2. Let $w_{1}$ and $w_{2}$ be odd positive integers. For $n \in \mathbb{Z}_{+}$, we have

$$
\sum_{k=0}^{n}\left(\begin{array}{l}
n \\
k
\end{array}\right) w_{1}^{k} w_{2}^{n-k} \mathcal{T}_{k, w_{2} \lambda} \gamma_{n-k, w_{1} \lambda}\left(2 w_{1}-1\right)=\sum_{k=0}^{n}\left(\begin{array}{l}
n \\
k
\end{array}\right) w_{2}^{k} w_{1}^{n-k} \mathcal{T}_{k, w_{1} \lambda} \gamma_{n-k, w_{2} \lambda}\left(2 w_{2}-1\right) .
$$

Letting $\lambda \rightarrow 0$ in Corollary 3.2 yields the identity

$$
\sum_{k=0}^{n}\left(\begin{array}{l}
n \\
k
\end{array}\right) w_{1}^{k} w_{2}^{n-k}\left(E_{n-k}\left(2 w_{1}-1\right)+T_{n-k}\right) T_{k}=\sum_{k=0}^{n}\left(\begin{array}{l}
n \\
k
\end{array}\right) w_{2}^{k} w_{1}^{n-k}\left(E_{n-k}\left(2 w_{2}-1\right)+T_{n-k}\right) T_{k} .
$$

Putting $w_{1}=1$ in Theorem 3.1 gives the identity

$$
2 \mathcal{T}_{n, w_{2} \lambda}\left(w_{2} x\right)=\sum_{k=0}^{n}\left(\begin{array}{l}
n \\
k
\end{array}\right) w_{2}^{k} \mathcal{T}_{k, \lambda}(x) \gamma_{n-k, w_{2} \lambda}\left(2 w_{2}-1\right)
$$

This may be rewritten as

$$
2 \mathcal{T}_{n, w_{2} \lambda}\left(w_{2} x\right)-2 w_{2}^{n} \mathcal{T}_{n, \lambda}(x)=\sum_{k=0}^{n-1}\left(\begin{array}{l}
n \\
k
\end{array}\right) w_{2}^{k} \mathcal{T}_{k, \lambda}(x) \gamma_{n-k, w_{2} \lambda}\left(2 w_{2}-1\right)
$$

By Theorem 2.3 and (3.3), we have the following theorem.

Theorem 3.3. Let $w_{2}$ be odd positive integer. For $n \in \mathbb{Z}_{+}$, we have

$$
\mathcal{T}_{n, w_{2} \lambda}\left(w_{2} x\right)=\sum_{k=0}^{n}\left(\begin{array}{l}
n \\
k
\end{array}\right) w_{2}^{k} \mathcal{T}_{k, \lambda}(x) \sum_{l=0}^{w_{2}-1}(-1)^{l}(2 l \mid \lambda)_{n-k} .
$$

We remark that (3.3) may also be used to give another proof of the distribution relation for degenerate tangent polynomials ([11, Theorem 5]): we observe that, since $(x \mid \lambda)_{n}$ is a homogeneous polynomial in $\lambda$ and $x$ of degree $n$, we have

$$
w_{2}^{-m} \gamma_{m, w_{2} \lambda}\left(2 w_{2}-1\right)=2 \sum_{i=0}^{w_{2}-1}(-1)^{i} w_{2}^{-m}\left(2 i \mid w_{2} \lambda\right)_{m}=2 \sum_{i=0}^{w_{2}-1}(-1)^{i}\left(\frac{2 i}{w_{2}} \mid \lambda\right)_{m} .
$$


Multiplying (3.3) by $w_{2}^{-n}$ and applying (2.6) then yields

$$
\begin{aligned}
2 w_{2}^{-n} \mathcal{T}_{n, w_{2} \lambda}\left(w_{2} x\right) & =\sum_{k=0}^{n}\left(\begin{array}{l}
n \\
k
\end{array}\right) w_{2}^{k-n} \mathcal{T}_{k, \lambda}(x) \gamma_{n-k, w_{2} \lambda}\left(2 w_{2}-1\right) \\
& =2 \sum_{k=0}^{n}\left(\begin{array}{l}
n \\
k
\end{array}\right) \mathcal{T}_{k, \lambda}(x) \sum_{i=0}^{w_{2}-1}(-1)^{i}\left(\frac{2 i}{w_{2}} \mid \lambda\right)_{n-k} \\
& =2 \sum_{i=0}^{w_{2}-1}(-1)^{i} \mathcal{T}_{n, \lambda}\left(\frac{2 i}{w_{2}}+x\right) .
\end{aligned}
$$

Therefore, we have the following theorem.

Theorem 3.4. Let $w_{2}$ be odd positive integer. For $n \in \mathbb{Z}_{+}$, we have

$$
\mathcal{T}_{n, w_{2} \lambda}\left(w_{2} x\right)=w_{2}^{n} \sum_{i=0}^{w_{2}-1}(-1)^{i} \mathcal{T}_{n, \lambda}\left(\frac{2 i}{w_{2}}+x\right) .
$$

Letting $\lambda \rightarrow 0$ in Theorem 3.4 gives the well-known distribution relation for usual tangent polynomials $\mathrm{T}_{\mathrm{n}}(\mathrm{x})$ (cf. [8, Theorem 2.3]).

\section{Distribution of zeros of the degenerate Euler-tangent mixed-type polynomials}

This section aims to demonstrate the benefit of using numerical investigation to support theoretical prediction and to discover new interesting pattern of the zeros of the degenerate Euler-tangent mixed-type polynomials $\gamma_{n, \lambda}(x)$. By using computer, the degenerate Euler-tangent mixed-type polynomials $\gamma_{n, \lambda}(x)$ can be determined explicitly. A few of them are

$$
\begin{aligned}
& \gamma_{0, \lambda}(x)=2 \\
& \gamma_{1, \lambda}(x)=x-1 \\
& \gamma_{2, \lambda}(x)=x^{2}-\lambda x+\lambda-1 \\
& \gamma_{3, \lambda}(x)=x^{3}-3 \lambda x^{2}+2 \lambda^{2} x-3 x-2 \lambda^{2}+3 \lambda+2 \\
& \gamma_{4, \lambda}(x)=x^{4}-6 \lambda x^{3}+11 \lambda^{2} x^{2}-6 x^{2}-6 \lambda^{3} x+18 \lambda x+6 \lambda^{3}-11 \lambda^{2}-12 \lambda+5 .
\end{aligned}
$$

We investigate the beautiful zeros of the $\gamma_{n, \lambda}(x)$ by using a computer. We plot the zeros of the degenerate Euler-tangent mixed-type polynomials $\gamma_{n, \lambda}(x)$ for $n=30, \lambda=1 / 10,1,2,3$, and $x \in \mathbb{C}$ (Figure 1).
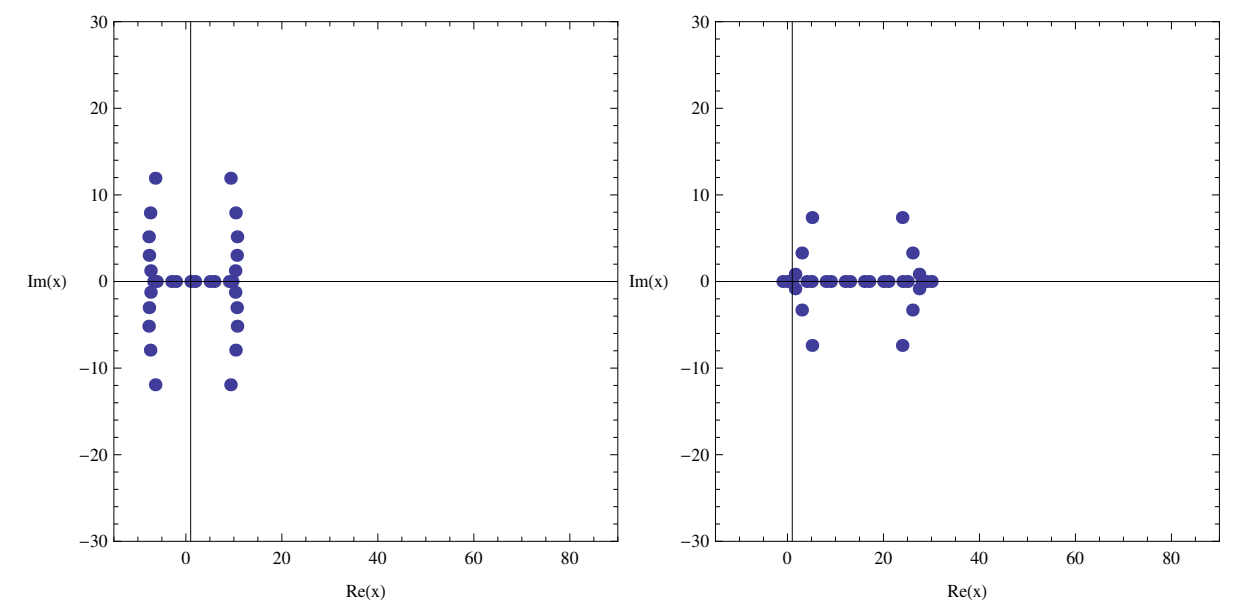

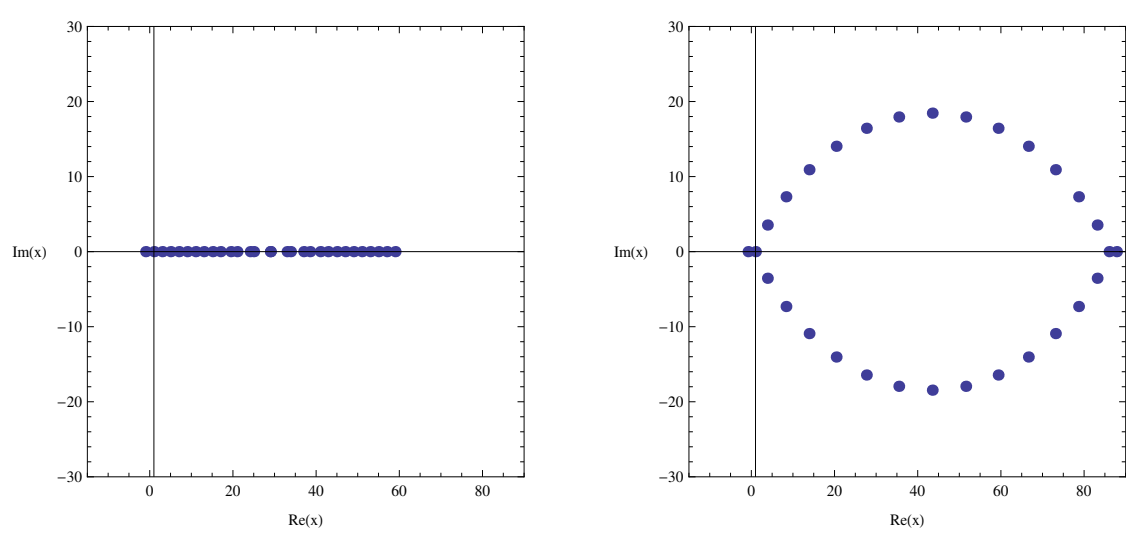

Figure 1: Zeros of $\gamma_{n, \lambda}(x)$.

In Figure 1 (top-left), we choose $n=30$ and $\lambda=1 / 10$. In Figure 1 (top-right), we choose $n=30$ and $\lambda=1$. In Figure 1 (bottom-left), we choose $n=30$ and $\lambda=2$. In Figure 1 (bottom-right), we choose $n=30$ and $\lambda=3$. Putting $\lambda=2$ in (2.1), the first few are

$$
\begin{aligned}
& \gamma_{0,2}(x)=2, \gamma_{1,2}(x)=x-1, \gamma_{2, \lambda}(x)=x^{2}-2 x+1, \gamma_{3,2}(x)=x^{3}-36 x^{2}+5 x \\
& \gamma_{4,2}(x)=x^{4}-12 x^{3}+38 x^{2}-12 x-15
\end{aligned}
$$

Stacks of zeros of $\gamma_{n, \lambda}(x)$ for $1 \leqslant n \leqslant 30$ from a 3-D structure are presented in Figure 2. In Figure 2 (left),
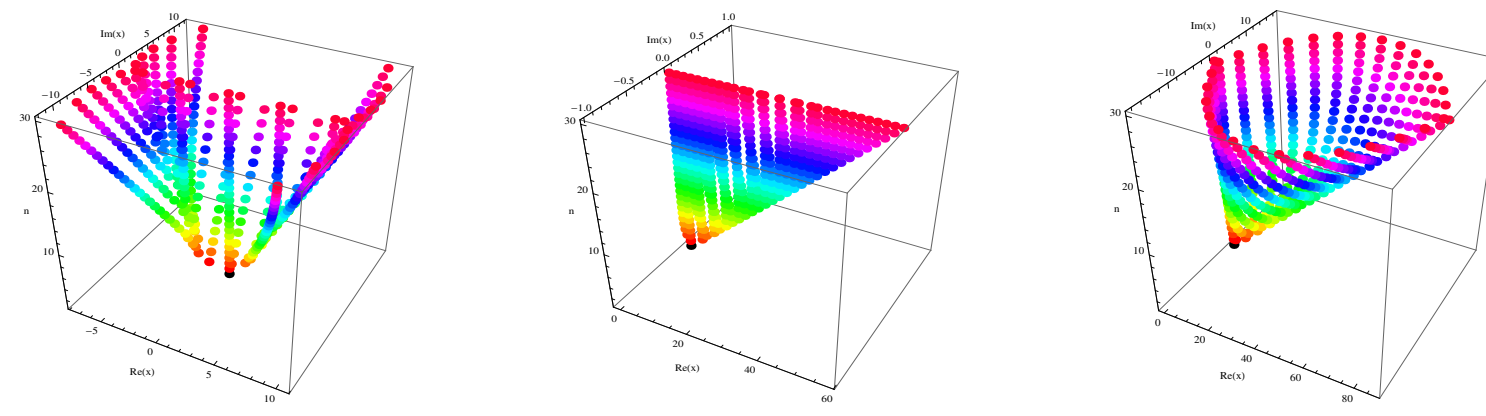

Figure 2: Stacks of zeros of $\gamma_{n, \lambda}(x)$ for $1 \leqslant n \leqslant 30$.

we choose $1 \leqslant n \leqslant 30$ and $\lambda=1 / 10$. In Figure 2 (middle), we choose $1 \leqslant n \leqslant 30$ and $\lambda=2$. In Figure 2 (right), we choose $1 \leqslant n \leqslant 30$ and $\lambda=3$. Our numerical results for approximate solutions of real zeros of $\gamma_{n, \lambda}(x)$ are displayed in Tables 1 and 2.

Plot of real zeros of $\gamma_{n, \lambda}(x)$ for $1 \leqslant n \leqslant 30$ structure are presented in Figure 3.
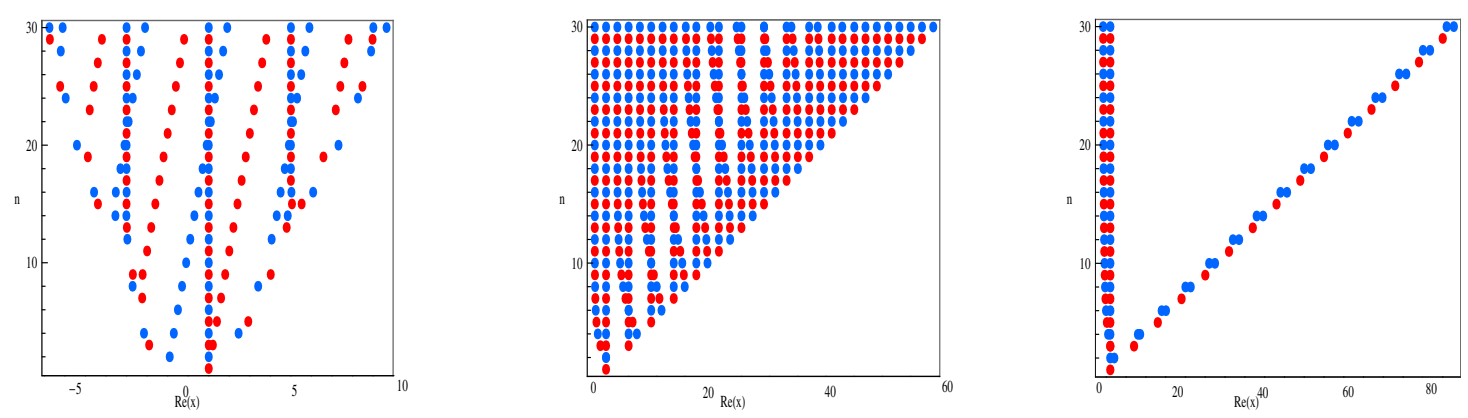

Figure 3: Real zeros of $\gamma_{n, \lambda}(x)$ for $\lambda=1 / 10,2,3$ and $1 \leqslant n \leqslant 30$. 
In Figure 3 (left), we choose $1 \leqslant n \leqslant 30$ and $\lambda=1 / 10$. In Figure 3 (middle), we choose $1 \leqslant n \leqslant 30$ and $\lambda=2$. In Figure 3 (right), we choose $1 \leqslant n \leqslant 30$ and $\lambda=3$. We observe a remarkably regular structure of the complex roots of the degenerate Euler-tangent mixed-type polynomials $\gamma_{n, \lambda}(x)$. We hope to verify a remarkably regular structure of the complex roots of the degenerate Euler-tangent mixed-type polynomials $\gamma_{n, \lambda}(x)$ (Table 1$)$.

Table 1: Numbers of real and complex zeros of $\gamma_{n, \lambda}(x)$.

\begin{tabular}{|c||cc|cc|cc|}
\hline \multirow{2}{*}{ degree $n$} & \multicolumn{3}{c|}{$\lambda=1 / 10$} & \multicolumn{2}{c|}{$\lambda=2$} & \multicolumn{2}{c|}{$\lambda=3$} \\
\cline { 2 - 7 } & real zeros & complex zeros & real zeros & complex zeros & real zeros & complex zeros \\
\hline \hline 1 & 1 & 0 & 1 & 0 & 1 & 0 \\
\hline 2 & 2 & 0 & 2 & 0 & 2 & 0 \\
\hline 3 & 3 & 0 & 3 & 0 & 3 & 0 \\
\hline 4 & 4 & 0 & 4 & 0 & 4 & 0 \\
\hline 5 & 3 & 2 & 5 & 0 & 3 & 2 \\
\hline 6 & 2 & 4 & 6 & 0 & 4 & 4 \\
\hline 7 & 3 & 4 & 7 & 0 & 3 & 4 \\
\hline 8 & 4 & 4 & 8 & 0 & 4 & 6 \\
\hline 9 & 5 & 4 & 9 & 0 & 3 & 8 \\
\hline 10 & 2 & 8 & 10 & 0 & 4 & 8 \\
\hline 11 & 3 & 8 & 11 & 0 & 3 & 4 \\
\hline 12 & 4 & 8 & 12 & 0 & & \\
\hline
\end{tabular}

Next, we calculated an approximate solution satisfying $\gamma_{n, \lambda}(x)$ for $x \in \mathbb{C}$. The results are given in Tables 2 and 3.

Table 2: Approximate solutions of $\gamma_{n, \lambda}(x)=0, \lambda=2, x \in \mathbb{R}$.

\begin{tabular}{|l|lllllll|}
\hline degree $\mathrm{n}$ & $\mathrm{x}$ \\
\hline \hline 1 & 1.0000 & & & & & \\
\hline 2 & $1.0000, \quad 1.0000$ & & & & & \\
\hline 3 & $0, \quad 1.0000, \quad 5.0000$ & & & & \\
\hline 4 & -0.46410, & 1.0000, & 5.0000, & 6.4641 & & & \\
\hline 5 & -0.70156, & 1.0000, & 5.0000, & 5.7016, & 9.0000 & & \\
\hline 6 & -0.83095, & 1.0000, & 5.0000, & 5.0000, & 9.0000, & 10.831 & \\
\hline 7 & -0.90381, & 1.0000, & 4.4418, & 5.0000, & 9.0000, & 10.462, & 13.000 \\
\hline
\end{tabular}

Table 3: Approximate solutions of $\gamma_{n, \lambda}(x)=0, \lambda=3, x \in \mathbb{R}$.

\begin{tabular}{|l|llll|}
\hline degree $\mathrm{n}$ & $\mathrm{x}$ & & & \\
\hline \hline 1 & 1.0000 & & & \\
\hline 2 & 1.0000, & 2.0000 & & \\
\hline 3 & 1.0000, & 1.0000, & 7.0000 & \\
\hline 4 & 0.46887, & 1.0000, & 8.0000, & 8.5311 \\
\hline 5 & 0.15248, & 1.000, & 13.000 & \\
\hline 6 & $-0.053737, \quad 1.000$, & 14.000, & 15.054 \\
\hline 7 & -0.19734, & 1.0000, & 19.000 & \\
\hline
\end{tabular}

By definition of the degenerate Euler-tangent mixed-type polynomials $\gamma_{n, \lambda}(x)$, we have the following theorem (see Tables 1 and 2). 
Theorem 4.1. For $\mathrm{n} \in \mathbb{Z}_{+}$, we have

$$
\gamma_{0, \lambda}(1)=2, \quad \gamma_{n, \lambda}(1)=0 .
$$

By numerical computations, we will make a series of the following conjectures.

Conjecture 4.2. Prove that $\gamma_{n, \lambda}(x), x \in \mathbb{C}$, has $\operatorname{Im}(x)=0$ reflection symmetry analytic complex functions. However, $\gamma_{n, \lambda}(x), x \in \mathbb{C}$, has not $\operatorname{Re}(x)=a$ reflection symmetry for $a \in \mathbb{R}$.

Using computers, many more values of $n$ have been checked. It still remains unknown if the conjecture fails or holds for any value $\mathrm{n}$ (see Figures 1, 2, and 3).

Conjecture 4.3. Prove that $\gamma_{n, \lambda}(x)=0$ has $n$ distinct solutions.

We find a counterexample of Conjecture 4.3. Let $\lambda=2$ and $n=6$. Then there are six numbers, $x_{i}(1=1,2,3,4,5,6)$ such that $\gamma_{6,2}\left(x_{i}\right)=0$. That is, we obtain $x_{1}=1, x_{2}=5, x_{3}=5, x_{4}=9, x_{5}=$ $5-\sqrt{34}, x_{6}=5+\sqrt{34}$. One may also observe that $\gamma_{2,2}(1)=0$ (see Table 2). Let $\lambda=3$ and $n=3$. Then there are three numbers, $x_{i}(1=1,2,3)$ such that $\gamma_{3,3}\left(x_{i}\right)=0$. That is, we obtain $x_{1}=1, x_{2}=1, x_{3}=7$ (see Table 3). Hence, Conjecture 4.3 is not true for all $n$. Letting $\lambda \rightarrow 0$, note that $\gamma_{2 n+1, \lambda}(x)=0$ has not $2 n+1$ distinct solutions for $n \in \mathbb{N}$. Using computers, many more values of $n$ and $\lambda$ have been checked. It still remains unknown if the conjecture fails or holds for any value $n$ and $\lambda$.

For fixed $\lambda$, since $n$ is the degree of the polynomial $\gamma_{n, \lambda}(x)$, the number of real zeros $R_{\gamma_{n, \lambda}(x)}$ lying on the real plane $\operatorname{Im}(x)=0$ is then $R_{\gamma_{n, \lambda}(x)}=n-C_{\gamma_{n, \lambda}(x)}$, where $C_{\gamma_{n, \lambda}(x)}$ denotes complex zeros. See Table 1 for tabulated values of $R_{\gamma_{n, \lambda}(x)}$ and $C_{\gamma_{n, \lambda}(x)}$.

Conjecture 4.4. Let $\lambda=2$. Prove that the numbers of complex zeros $C_{\gamma_{n, \lambda}(x)}$ of $\gamma_{n, \lambda}(x), \operatorname{Im}(x) \neq 0$ are $C_{\gamma_{n, \lambda}(x)}=$ 0 .

Conjecture 4.5. Putting $\lambda=3$, prove that the numbers of complex zeros $C_{\gamma_{n, \lambda}(x)}$ of $\gamma_{n, \lambda}(x), \operatorname{Im}(x) \neq 0$ are

$$
\mathrm{C}_{\gamma_{n, \lambda}(x)}=2\left[\frac{\mathrm{n}-3}{2}\right], \text { for } n \geqslant 3 \text {, }
$$

where [.] denotes taking the integer part.

Finally, we shall consider the more general problems. How many zeros does $\gamma_{n, \lambda}(x)$ have? Find the numbers of complex zeros $C_{\gamma_{n, \lambda}(x)}$ of $\gamma_{n, \lambda}(x), \operatorname{Im}(x) \neq 0$. Since $n$ is the degree of the polynomial $\gamma_{n, \lambda}(x)$, the number of real zeros $R_{\gamma_{n, \lambda}(x)}$ lying on the real plane $\operatorname{Im}(x)=0$ is then $R_{\gamma_{n, \lambda}(x)}=n-C_{\gamma_{n, \lambda}(x)}$, where $C_{\gamma_{n, \lambda}(x)}$ denotes complex zeros. See Table 1 for tabulated values of $R_{\gamma_{n, \lambda}(x)}$ and $C_{\gamma_{n, \lambda}(x)}$. The author has no doubt that investigations along this line will lead to a new approach employing numerical method in the research field of the degenerate Euler-tangent mixed-type polynomials $\gamma_{n, \lambda}(x)$ to appear in mathematics and physics. The reader may refer to $[4,7,10]$ for the details.

\section{Acknowledgment}

This work was supported by the National Research Foundation of Korea (NRF) grant funded by the Korea government (MEST) (No. 2017R1A2B4006092).

\section{References}

[1] M. Açikgöz, D. Erdal, S. Araci, A new approach to q-Bernoulli numbers and q-Bernoulli polynomials related to qBernstein polynomials, Adv. Difference Equ., 2010 (2010), 9 pages. 1

[2] L. Carlitz, Degenerate Stirling, Bernoulli and Eulerian numbers, Utilitas Math., 15 (1979), 51-88. 1

[3] D. V. Dolgy, T. Kim, S.-H. Rim, S. H. Lee, Symmetry identities for the generalized higher-order q-Bernoulli polynomials under $S_{3}$ arising from p-adic Volkenborn ingegral on $\mathbb{Z}_{\mathrm{p}}$, Proc. Jangjeon Math. Soc., 17 (2014), 645-650. 
[4] J. Y. Kang, C. S. Ryoo, A research on the some properties and distribution of zeros for Stirling polynomials, J. Nonlinear Sci. Appl., 9 (2016), 1735-1747. 4

[5] T. K. Kim, Barnes' type multiple degenerate Bernoulli and Euler polynomials, Appl. Math. Comput., 258 (2015), $556-$ 564.

[6] F. Qi, D. V. Dolgy, T. Kim, C. S. Ryoo, On the partially degenerate Bernoulli polynomials of the first kind, Glob. J. Pure Appl. Math., 11 (2015), 2407-2412. 1

[7] C. S. Ryoo, Calculating zeros of the second kind Euler polynomials, J. Comput. Anal. Appl., 12 (2010), 828-833. 4

[8] C. S. Ryoo, A note on the symmetric properties for the tangent polynomials, Int. J. Math. Anal. (Ruse), 7 (2013), $2575-$ 2581. $1,1,3,3$

[9] C. S. Ryoo, A note on the tangent numbers and polynomials, Adv. Stud. Theoret. Phys., 7 (2013), 447-454. 1

[10] C. S. Ryoo, A numerical investigation on the zeros of the tangent polynomials, J. Appl. Math. Inform., 32 (2014), 315-322. 4

[11] C. S. Ryoo, Notes on degenerate tangent polynomials, Glob. J. Pure Appl. Math., 11 (2015), 3631-3637. 1, 1, 3

[12] P. T. Young, Degenerate Bernoulli polynomials, generalized factorial sums, and their applications, J. Number Theory, 128 (2008), 738-758. 1 